\title{
Application of Two Dimensional Haar Transforms and Fast Haar functions to Analysis High OrderSystemin Time and Frequency Domain
}

\author{
Joon-Hoon Park \\ Korea National University of Transportation, Korea \\ jhpark@ut.ac.kr
}

\begin{abstract}
In this paper, a method for analysis high order transfer function system via two dimensional Haar transforms is proposed. A high order transfer function system can be transformed and simplified to a low order system that has same system response. For these, fast Haar function seriesthat is a complete set of orthogonal rectangular functions similar in several respects of the Walsh functionsand two dimensional Haar transform algorithm are applied. And the results are simulated in time and frequency domain. The suggested method is more efficient and convenient for simplification of high order system.
\end{abstract}

Keywords: high order transfer function system, simplification, Haar functions, two dimensional transforms

\section{Introduction}

Many orthogonal functions used frequently in mathematical and engineering fields are defined as solution of control system analysis and design such as ordinary and partial differential equation of linear and non-linear systems. Also, it is known that high order transfer function system open has insignificant poles that have little effect on the system response.In this case, the less significant poles can be disregarded and the systemis simplified as low order system. Therefore, a method for simplification of high order system will be useful to analysis and design of control system. The Haar functions were introduced by Alfred Haar rather earlier than the Walsh functions that are complete set orthogonal rectangular functions. TheHaar functions also a set of orthogonal functions that have essentially only two values and they provide an expansion of a given continuous function. And the fast Haar functions transform a system rapidly and have some computational advantages in certain areas of communication, digital filtering and control system.The objectives of this paper are to propose an algorithm of reducing system order such that has computational advantages.For these purpose, the fast Haar functions and two dimensional Haar transform are applied to simplify high order transfer function in this paper. The developed algorithm and its results are simulated and examined in time and frequency domain for system response analysis. The presented methodin this paper is useful for system simplification, analysis and design. The Haar functions form an orthogonal and orthonormal system of periodic square waves. The amplitude values of these square waves do not have uniform value, but have limited set of values. If we consider the time base to be defined as $0 \leq t \leq 1$ then, the Haar functions is described as follows:

$$
\operatorname{Har}(0, t)=1 \text { for } 0 \leq t \leq 1
$$




$$
\begin{aligned}
& \operatorname{Har}(1, t)=\left\{\begin{array}{cc}
1 & \text { for } 0 \leq t \leq \frac{1}{2} \\
-1 & \text { for } \frac{1}{2} \leq t \leq 1
\end{array}\right. \\
& \operatorname{Har}(2, t)=\left\{\begin{array}{rc}
\sqrt{2} & \text { for } 0 \leq t \leq \frac{1}{4} \\
-\sqrt{2} & \text { for } \frac{1}{4} \leq t \leq \frac{1}{2} \\
0 & \text { for } \frac{1}{2} \leq t \leq 1
\end{array}\right. \\
& \operatorname{Har}(3, t)=\left\{\begin{array}{cc}
0 & \text { for } 0 \leq t \leq \frac{1}{2} \\
\sqrt{2} & \text { for } \frac{1}{2} \leq t \leq \frac{3}{4} \\
-\sqrt{2} & \text { for } \frac{3}{4} \leq t \leq 1
\end{array}\right. \\
& \operatorname{Har}\left(2^{p}+n, t\right) \\
& =\left\{\begin{array}{rc} 
& \sqrt{2^{p}} \quad \text { for } \frac{n}{2^{p}} \leq t \leq \frac{n+\frac{1}{2}}{2^{p}} \\
-\sqrt{2^{p}} & \text { for }\left(n+\frac{1}{2}\right) / 2^{p} \leq t \leq(n+1) / 2^{p(1.2)} \\
0 & \text { elsewhere }
\end{array}\right.
\end{aligned}
$$

We can express the Haar functions using different definition for the series as follows:

$$
=\left\{\begin{array}{ccc}
\sqrt{2^{i}} & \text { for } \quad \frac{j-1}{2^{i}} \leq t \leq\left(j-\frac{1}{2}\right) / 2^{i} \\
-\sqrt{2^{i}} & \text { for }\left(j-\frac{1}{2}\right) / 2^{i} \leq t \leq \frac{j}{2^{i}} \\
0 & \text { elsewhere }
\end{array}\right.
$$

Then, the Haar functions can be referred to by order jand degree I as well as time t. The degree $i$ denotes a subset having the same number of zero crossing in given width $\frac{1}{2^{i}}$ The order $\mathrm{j}$ gives the position of the function within this subset. All members of the subset with the same degree are obtained by shifting the first member along the axis by an amount proportional to this order.In figure 1, the first eight Haar functions are shown.[1][2] 


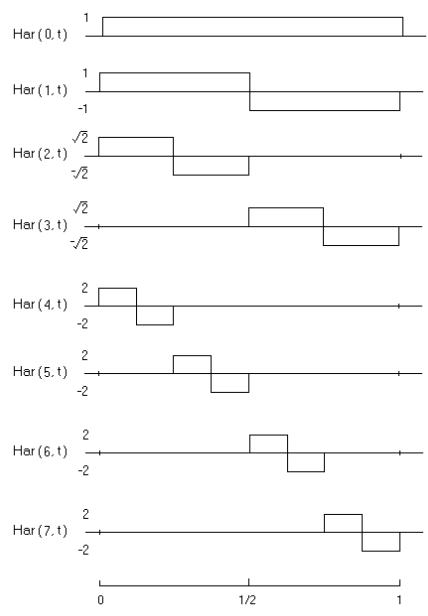

Figure 1 The First Eight Haar Functions

\section{Haar Function Series Expansion}

As the equation (1.3), we can define that the Haar functions are orthogonal, so that we can write the Haar functions as

$$
\int_{0}^{t} \operatorname{Har}(m, t) \operatorname{Har}(n, t)=\left\{\begin{array}{ll}
1 & \text { for } n=m \\
0 & \text { for } n \neq m
\end{array}(2.1)\right.
$$

A function $f(t)$ is absolutely integral on then it can be expanded as an infinite series in term of the Haar functions.

$$
\begin{aligned}
& f(t)=c_{0} \operatorname{Har}_{0}(t)+c_{1} \operatorname{Har}_{1}(t)+c_{2} \operatorname{Har}_{2}(t)+\cdots \\
& =\sum_{i=0}^{\infty} c_{i} \operatorname{Har}_{i}(t)(2.2)
\end{aligned}
$$

Where $c_{i}$ is the $i$ th sequentially ordered coefficient of the Haar functions expansion of function $f(t)$ and $h_{i}$ is the $i$ th ordered Haar functions. The coefficient of the Haar functions expansion is given as equation (2.2).

$$
c_{i}=\int_{0}^{1} f(t) \operatorname{Har}(i, t) d t
$$

To obtain the coefficients of Haar transforms, mean square error $\varepsilon$ must be minimized and partial differential equation for $\mathrm{c}_{i}$ set to be zero.

$$
\begin{aligned}
& \varepsilon=\int_{0}^{1}\left[f(t)-\sum_{i=0}^{m-1} F_{i} R H a r(i, t)\right]^{2} d t \\
& \frac{\partial \varepsilon}{\partial F_{i}}=2 \int_{0}^{1}\left[f(t)-\sum_{i=0}^{m-1} F_{i} R H a r(i, t)\right] R H a r(i, t) d t=0
\end{aligned}
$$

For example, if $f(t)=t, f(t)$ can be expanded and approximated using Haar functions and the result is shown in figure 2.[3] 


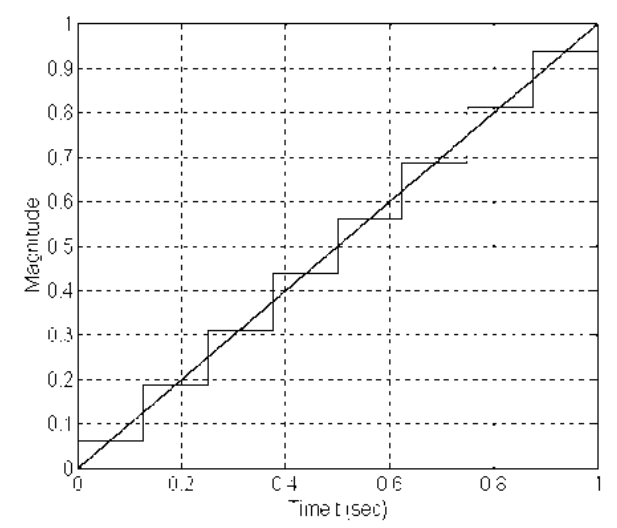

Figure 2 Haar Transform of $f(t)=t$ with $n=8$

With regard to the convergence characteristic property of expansion, the Haar functions are excellent to the Walsh functions because the Walsh functions are expanded with divergences at a given point but the Haar functions are not occurred in the case of divergences. And the coefficients of expansion are proportional to the difference in the mean value of $f(t)$ over the adjacent partial periods and intervals. The Haar operational matrix can be written as follows:

$$
\int_{0}^{t} \operatorname{Har}(i, \tau) d \tau=\operatorname{PHar}(i, t)
$$

The operational matrix $P$ is defined as equation (2.7) and as an example, $P_{(2 x 2)}$ is shown in equation (2.8).

$$
\begin{aligned}
& P_{(m \times m)}=\left[\begin{array}{cc}
P_{\left(\frac{m}{2} \times \frac{m}{2}\right)} & -\frac{1}{\sqrt{2}} m^{-\frac{3}{2}} \operatorname{Har}_{\left(\frac{m}{2} \times \frac{m}{2}\right)} \\
\frac{1}{\sqrt{2}} m^{-\frac{3}{2}} \operatorname{Har}_{\left(\frac{m}{2} \times \frac{m}{2}\right)} & 0_{\left(\frac{m}{2} \times \frac{m}{2}\right)}
\end{array}\right] \\
& P_{(2 \times 2)}=\left[\begin{array}{cc}
\frac{1}{2} & -\frac{1}{4} \\
\frac{1}{4} & 0
\end{array}\right]
\end{aligned}
$$

If $f(t)=t^{2}$, then $f(t)$ can be expressed using Haar transform and operational matrixPas follows:

$$
\begin{aligned}
& f(t)=t^{2}=2 \int_{0}^{t} c_{i} \operatorname{Har}_{i}(\tau) d \tau=2 c \times P \times H a r(t) \\
& f(t)=0.3359 \operatorname{Har}_{0}(t)-0.25 \operatorname{Har}_{1}(t)-0.0442 \operatorname{Har}_{2}(t)-0.1326 \operatorname{Har}_{3}(t) \\
& -0.0078 \operatorname{Har}_{4}(t)-0.023 \operatorname{Har}_{5}(t)-0.039 \operatorname{Har}_{6}(t)-0.0547 \operatorname{Har}_{7}(t)
\end{aligned}
$$

In figure 3 and 4, the results of Haar transform with the operational matrix and the first eight integration of Haar functions respectively. 


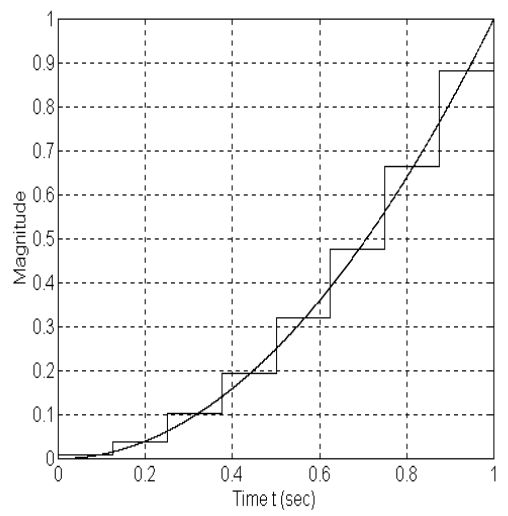

Figure 3 Haar Transform of $f(t)=t^{2}$ with Operational Matrix $P$

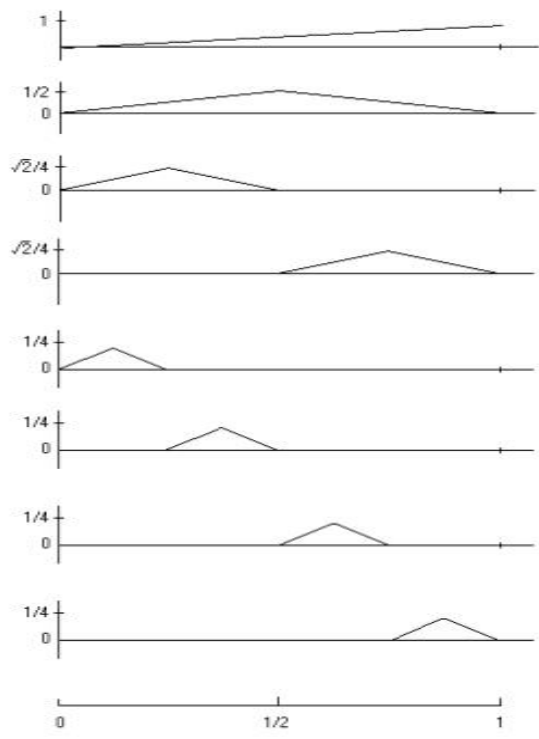

Figure 4 The First Eight Integration of Haar Functions

\section{Discrete and Fast Haar Transform}

As more and more digital technology and computational ability are increasing and spreading widely, the transforms of orthogonal functions have been becoming a practical method and toolin communication, signal processing, control systems. At the beginning those transfer algorithms were applied only to the Fourier analysis but have now been extended to various orthogonal functions and transforms. Furthermore in case that if numerical and mathematical process are difficult or impossible, we can apply the orthogonal transforms to those problems instead of mathematical methods. As we know, the orthogonal transforms include the Walsh, Hadamard, Haar and various other transforms.Let's consider the discrete Haar transform from equation (2.2) then it can be obtained as follows:[4]

$$
\begin{aligned}
& {H^{*}}_{m}=\sum_{i=0}^{\mathrm{m}-1} C_{i} \operatorname{Har}(m, i) \\
& C_{i}=\frac{1}{m} \sum_{n=0}^{m-1} \operatorname{Har}(i, m) H^{*}{ }_{m}(3.2)
\end{aligned}
$$


where $i, m=0,1, \ldots, n-1$. We can write the equation (3.1) in matrix form. In equation (3.1), $H_{m}^{*}$ is discrete sampling results of $f(t)$ and $C_{i}$ is coefficient of discrete Haar transforms.

$$
\begin{aligned}
& H^{*}=\left[H_{0}^{*}, H_{1}^{*}, \ldots, H_{m-1}^{*}\right]^{T} \mathrm{C}=\left[C_{0}, C_{1}, \ldots, C_{m-1}\right]^{T}(3.3) \\
& \operatorname{Har}_{i}=\left[\operatorname{Har}_{i 0} \operatorname{Har}_{i 1} \operatorname{Har}_{i 2} \ldots \operatorname{Har}_{i(m-1)}\right]^{T} \\
& H^{*}=\operatorname{Har}^{T} C \\
& C=\frac{1}{m} \operatorname{HarH}^{*}(3.6)
\end{aligned}
$$

where $H^{*}$ and $\operatorname{Har}^{T}$ are the direct and transposed Haar function matrices.

$$
H_{8}^{*}=\left[\begin{array}{cccccccr}
1 & 1 & 1 & 1 & 1 & 1 & 1 & 1 \\
1 & 1 & 1 & 1 & -1 & -1 & -1 & -1 \\
\sqrt{2} & \sqrt{2} & -\sqrt{2}-\sqrt{2} & 0 & 0 & 0 & 0 \\
0 & 0 & 0 & 0 & \sqrt{2} & \sqrt{2} & -\sqrt{2} & \sqrt{2} \\
2 & -2 & 0 & 0 & 0 & 0 & 0 & 0 \\
0 & 0 & 2 & -2 & 0 & 0 & 0 & 0 \\
0 & 0 & 0 & 0 & 2 & -2 & 0 & 0 \\
0 & 0 & 0 & 0 & 0 & 2 & -2
\end{array}\right]
$$

The discrete Haar transform matrices are stated in equation (3.7). They are not symmetric, thus the discrete Haar transform needs segmented transform operations for transform processing. The discrete transform matrix is the product of diagonal matrix that contains multiples of $\sqrt{2}$. To obtain computational efficiency and improved processing algorithm, the factorial calculation method is applied to the original Haar transform. And then fast Haar transform is defined. The fast Haar transform gives the fastest linear transform because of its simplicity and particularly values. The computationalflow graph for fast Haar transform with 8 nodes is shown in figure 5 and inverse fast Haar transform is shown in figure 6. In figures, the solid line stands for addition and dotted line is for subtraction at each node. The total number of addition and subtraction can be stated as equation (3.8). And the transform time is depends on the number of terms N.The fast transform has less complicated computational lines comparing with the original Haar transform. Therefore fast Haar transform is better than the original Haar transform in computational time and efficiency.[5][6]

$$
\mathrm{N}+\frac{N}{2}+\frac{N}{4}+\cdots+2=2(N-1)
$$

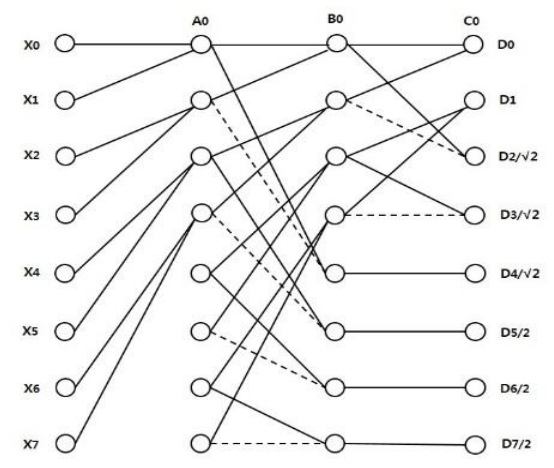

Figure 5. Computational Flow Graph of the Fast Haar Transform with $n=8$ 


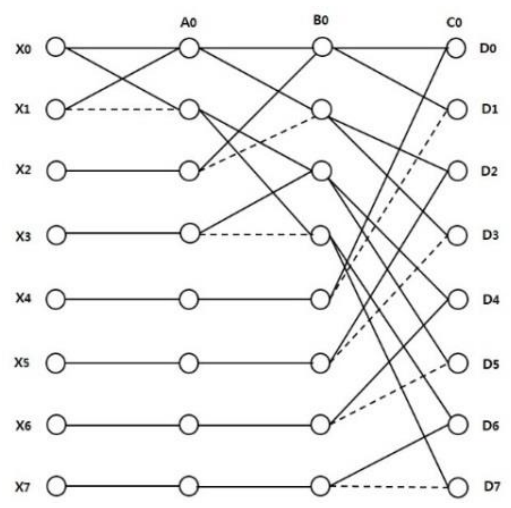

Figure 6 Computational Flow Graph of the Inverse Fast Haar Transform with $n=8$

\section{High Order System Analysis via Two Dimensional Transform}

Let's consider forward path transfer function as follows:

$$
\mathrm{G}(\mathrm{s})=\mathrm{K} \frac{1+b_{1} s+b_{2} s^{2}+\cdots+b_{m} s^{m}}{1+a_{1} s+a_{2} s^{2}+\cdots+a_{n} s^{n}}(4.1)
$$

Then we can obtain highorder transfer function $M_{H}(s)$ in equation (4.2)andwe can set and assume a loworder transfer function $M_{L}(s)$ as equation (4.3).

$$
\begin{aligned}
& M_{H}(\mathrm{~s})=\mathrm{K} \frac{1+b_{1} s+b_{2} s^{2}+\cdots+b_{m} s^{m}}{1+a_{1} s+a_{2} s^{2}+\cdots+a_{n} s^{n}} \\
& M_{L}(\mathrm{~s})=\mathrm{K} \frac{1+c_{1} s+c_{2} s^{2}+\cdots+c_{q} s^{q}}{1+d_{1}+d_{2} s^{2}+\cdots+d_{p} s^{p}}(4.3)
\end{aligned}
$$

where $n \geq m, n \geq p \geq q$. In this case, the zero frequency gain $\mathrm{K}$ of the two transfer functions is the same. And $s=j w$ is applied to above equations. And the zero frequency gain $\mathrm{K}$ of the two transfer functions is the same. Thus, we can obtain the criterion of finding the loworder $M_{L}(s)$, given $M_{H}(s)$, is that the following relation should be satisfied (4.4).[7][8]

$$
\frac{\left|M_{H}(j w)\right|^{2}}{\left|M_{L}(j w)\right|^{2}}=1(4.4)
$$

Equating both side of equation (4.4) and satisfying the condition, similar relationships can be obtained for coefficients of equation (4.2) and (4.3). To obtain and determine the coefficients of low ordertransfer function system, we can apply the two dimensional Haar transform to the high order transfer function system. The two dimensional Haar transform is very useful for control system analysis including high order transfer function system. The array of two dimensional Haar transform can be written as equation (4.5).

$$
X_{m, n}=\frac{1}{N^{2}} \sum_{i=0}^{N-1} \sum_{j=0}^{N-1} x_{i, j} \operatorname{Har}\left(n, \frac{i}{N}\right) \operatorname{Har}\left(m, \frac{j}{N}\right)
$$

The two dimensional transform is performed in two processing steps. First transform is carried out for the variables.

$$
X_{m, j}=\sum_{i=0}^{N-1} x_{i, j} \operatorname{Har}\left(m, \frac{i}{N}\right)(4.6)
$$


And then second one dimensional transform is accomplished with each column of transformed array for the variables.

$$
X_{m, n}=\sum_{j=0}^{N-1} x_{m, j} \operatorname{Har}\left(m, \frac{j}{N}\right)(4.7)
$$

This transform algorithm is basically single dimensional operation but the processing data is constructed from consecutive subsets of second step and two dimensional transform. Figure 7 shows basic two dimensional transform concept and in figure 8 , series of two dimensional Haar transform is shown.

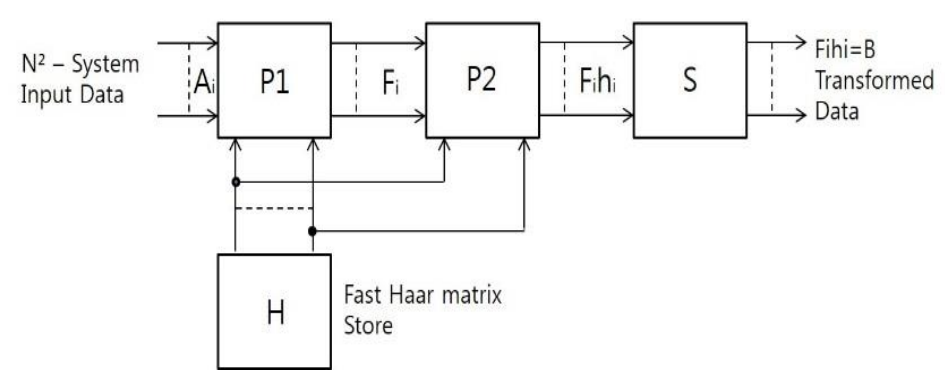

Figure 7 Concept of Two Dimensional Transform
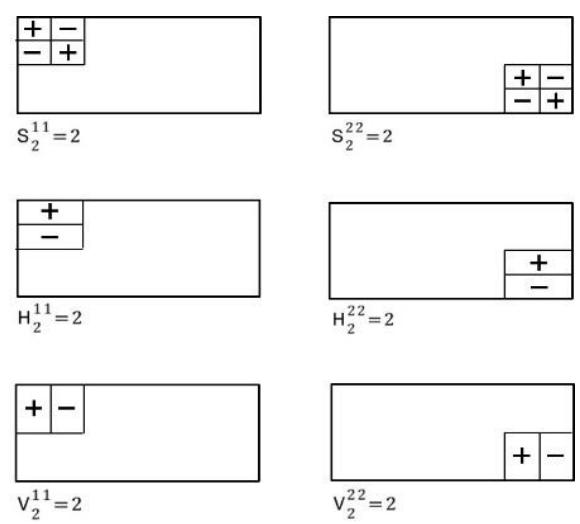

\section{Figure 8 Series of the Two Dimensional Haar Transform}

In figure $8, S_{n}^{i j}$ is stated as equation (4.8) and as the same manner $H_{n}^{i j}$ and $V_{n}^{i j}$ are defined respectively.

$$
S_{n}^{i j}(\mathrm{x}, \mathrm{y})=\operatorname{Har}_{y}(n, i) \operatorname{Har}_{x}(n, i)(4.8)
$$

\section{Simulation}

Case 1:Consider a control system that has forward path transfer function and feedback control path. The system can be described by equation (5.1).[9]

$$
\mathrm{G}(\mathrm{s})=\frac{1}{0.25 s^{3}+0.7 s^{2}+1.15 s}, H(s)=1
$$

From the above equations, we have third order transfer function of the given control system. 


$$
M_{H 1}(s)=\frac{1}{1+1.15 s+0.7 s^{2}+0.25 s^{3}}
$$

To compare the results, we use numerical method to get reduced order system. $M_{L N I}(s)$ is low order transfer function by numerical method.

$$
M_{L N 1}(s)=\frac{1}{1+0.426 s+0.23 s^{2}}(5.3)
$$

And we can apply the two dimensional Haar transform that is suggested in this paper to get the loworder transfer function $M_{L H T I}(s)$ from the original high order transfer function $M_{H I}(\mathrm{~s})$.

$$
M_{L H T 1}(s)=\frac{1}{1+0.48 s+0.21 s^{2}}(5.4)
$$

The time domain response results are shown in figure 9 to 11 . The high order system analysis between the original third order system and the numerical secondorder system is shown in figure 9. The dashed line is for the original thirdorder system and the dashdotted graph displays the numerical secondorder system.

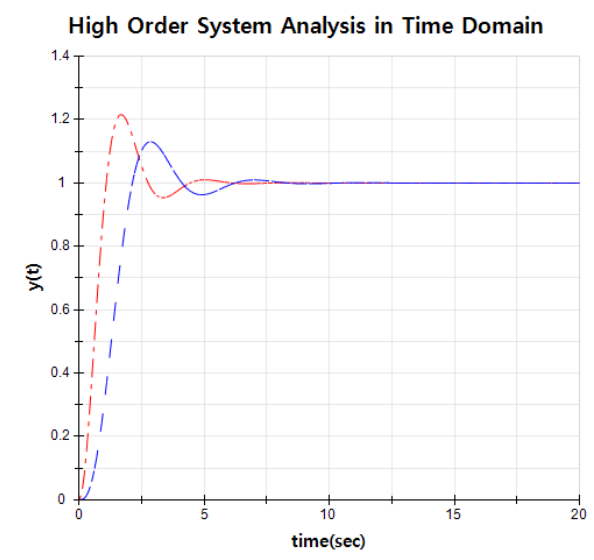

Figure 9 Time Domain Analysis for $M_{H 1}(s)$ and $M_{L 1}(s)$

And figure 10 shows analysis between the original thirdorder system and the proposed secondorder system. In the figure, dotted line stands for the proposed secondorder system. And all three simulation analysis results are shown in figure 11.

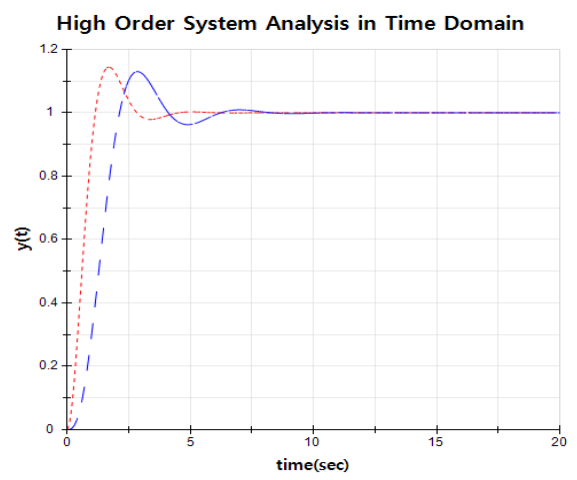

Figure 10 Time Domain Analysis for $M_{H 1}(s)$ and $M_{H T 1}(s)$ 


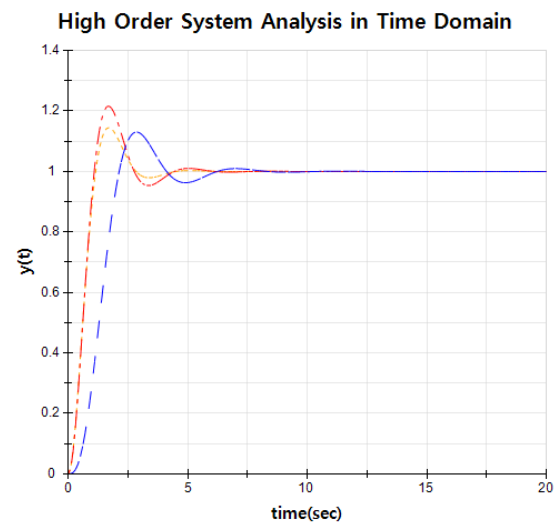

Figure 11 Time Domain Analysis for $M_{H 1}(s), M_{L 1}(s)$ and $M_{H T 1}(s)$

Case 2: Now we try to simulate the proposed method in frequency domain as the same process in case 1. The high order transfer function is stated as equation (5.5).

$$
M_{H 2}(s)=\frac{1}{1+1.5 s+1.2 s^{2}+0.2 s^{3}}(5.5)
$$

To compare the results, we also usenumerical method to obtain the $M_{L N 2}(s)$ that is low order transfer function.

$$
M_{L N 2}(s)=\frac{1}{1+1.6 s+1.13 s^{2}}(5.6)
$$

And we can apply the two dimensional Haar transform to the original high order system to determine $M_{L H T 2}(s)$ in equation (5.7).

$$
M_{\text {LHT2 }}(s)=\frac{1}{1+1.41 s+1.17 s^{2}}(5.7)
$$

In figure 12 to 14 , the frequency domain response results are shown.The high order system analysis between the original third order system and the numerical secondorder system is shown in figure 12. The dashed line is for the original thirdorder system and the dash-dotted graph displays the numerical secondorder system.

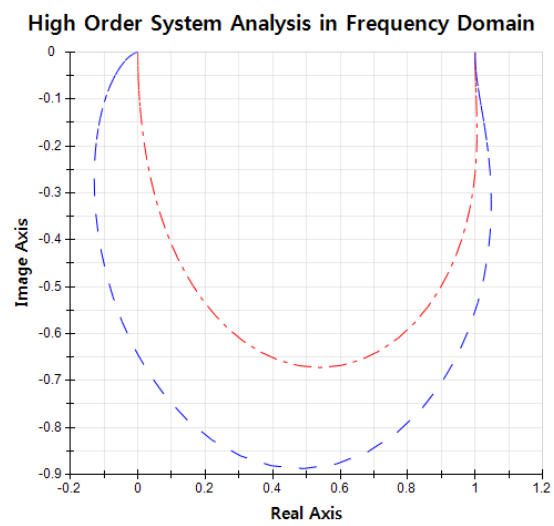

Figure 12 Frequency Domain Analysis for $M_{H_{2}}(s)$ and $M_{L 2}(s)$

And figure 13 shows analysis between the original thirdorder system and the proposed 
secondorder system. In the figure, solid line stands for the proposed secondorder system. And all three simulation analysis results are shown in figure 14.

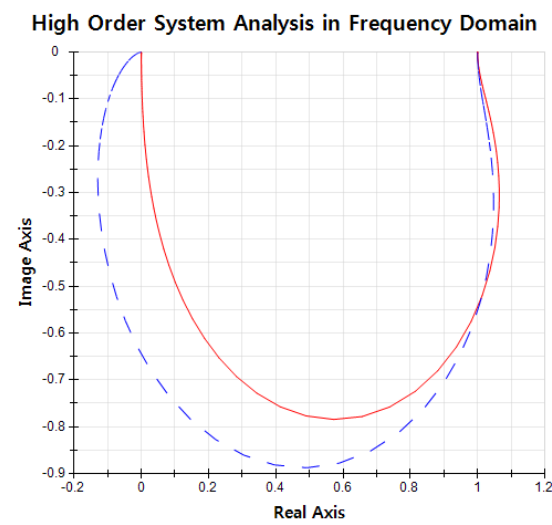

Figure 13 Frequency Domain Analysis for $M_{H 2}(s)$ and $M_{H T 2}(s)$

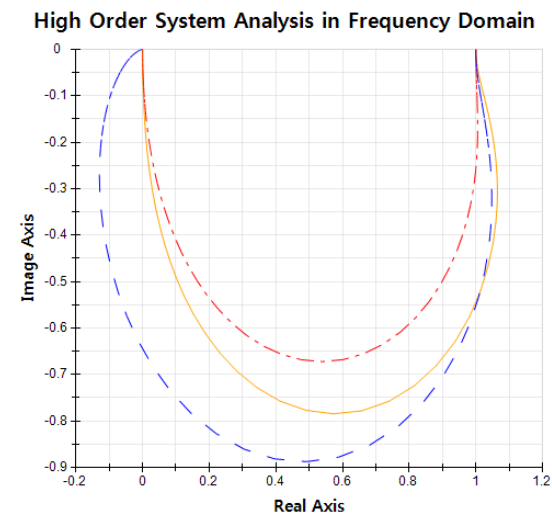

Figure 14 Frequency Domain Analysis for $M_{H 2}(s), M_{L 2}(s)$ and $M_{H T 2}(s)$

\section{Conclusions}

In this paper, the two dimensional Haar transform isproposed for high order system analysis.And the simulation and analysis results are examined in time and frequency domain. Reducinghigh order system to low order system is very useful and meaningful because high order transfer function system often contains insignificant solutions that have less important influences on the system responses. In figure 9, 10 and 11, the system responses and results such as delay and setting time, damping ratio show almost same results between $M_{L N I}(s)$ and $M_{L H T I}(s)$, but there is a slight difference in the maximum overshoot. As compared with the original high order system, the difference of maximum overshoot of $M_{L N I}(s)$ is about $25 \%$, while $M_{L H T I}(s)$ is about $15 \%$. Thus, $M_{L H T I}(s)$ is shown better system response than $M_{L N I}(s)$ and $M_{L H T I}(s)$ isapproached to $M_{H I}(s)$ more closely. In this case,the rising time of $M_{L H T I}(s)$ is shorter than $M_{H 1}(s)$ so that its maximum overshoot must be greater than $M_{H 1}(s)$. In figure 12 to 14 , thedifferencesin frequency response between $M_{H 2}(s), M_{L N 2}(s)$ and $M_{L H T 2}(s)$ are shown. As shown in figures, the $M_{L H T 2}(s)$ display better results than $M_{L N 2}(s)$ but the frequency response gap isslightly big. From computational efficiency point of view, the two dimensional Haar transform reduce and save time. Overall the result of proposed method is better than the result of numerical 
method in terms of accuracy. Thus, the presented method for analysis high order transfer functionsystem in this paper is valuable and useful.

\section{Acknowledgement}

"This paper is a revised and expanded version of a paper entitled<Approximation of High Order Characteristic Equation using Discrete Haar Transforms> presented atThe $2^{\text {nd }}$ International Conference on AICT2014, Hungry."

\section{References}

[1.] K. G. Beauchamp, "Walsh Functions and Their Applications", Academic Press, (1975).

[2.] J. H. Park, "Trajectory Optimization for Large Scale Systems via Block Pulse Functions and Transformations", Int. J. of Control and Automation, vol. 5, no. 4, (2013), pp. 39-48.

[3.] Z. H. Jiang, "Block Pulse Functions and Their Applications in Control Systems", Springer-Verlag, (1992).

[4.] J. H. Park, "System Order Reduction of Transfer Function via Haar Function in Time Domain", Int. J. of ACT, vol. 3, no. 1, (2014), pp. 84-87.

[5.] J. H. Park, "Transfer Function Approximation using Rationalized Haar Transform in Frequency Domain", Int. J. of CA, vol. 7, no. 4, (2014), pp. 247-258.

[6.] J. H. Park and R. D. Oh, "Transform of characteristic equation using fast rationalized Haar functions", $4^{\text {th }}$ International Work Shop on Control and Utilization of Sensors (2014).

[7.] J. H. Park and R. D. Oh, "Transform algorithm for system order simplification using block pulse functions and operational matrix", $4^{\text {th }}$ International Work Shop on Control and Utilization of Sensors, (2014).

[8.] B. C. Kuo, “Automatic Control System”, $9^{\text {th }}$ edition, Wiley, (2009).

[9.] J. H. Park, "Approximation of High Order Characteristic Equation using Discrete Haar Transforms", The $2^{\text {nd }}$ International Conference on AICT, (2014). 